\title{
PROBABILISTIC ANALYSIS OF THE GENERALISED ASSIGNMENT PROBLEM
}

\author{
Martin Dyer* \\ School of Computer Studies, University of Leeds, Leeds, U.K. \\ and \\ Alan Frieze ${ }^{\dagger}$ \\ Department of Mathematics, Carnegie-Mellon University, \\ Pittsburgh, U.S.A.
}

December 19, 1999

\begin{abstract}
We analyse the generalised assignment problem under the assumption that all coefficients are drawn uniformly and independently from $[0,1]$. We show that such problems can be solved exactly with high probability, in a well-defined sense. The results are closely related to earlier work of Lueker, Goldberg and Marchetti-Spaccamela and ourselves.
\end{abstract}

\section{Introduction}

We are concerned here with the generalised assignment problem:

$$
\begin{array}{llll}
\text { Maximise } & z=\sum_{i=1}^{m} \sum_{j=1}^{n} c_{i j} x_{i j} & & \\
\text { subject to } & \sum_{j=1}^{n} a_{i j} x_{i j} & \leq b_{i}=\beta_{i} n & (i=1,2, \ldots, m) \\
& \sum_{i=1}^{m} x_{i j} & =1 & (j=1,2, \ldots, n) \\
\text { and all } & x_{i j} \in\{0,1\} . & &
\end{array}
$$

This problem has applications in machine scheduling and elsewhere. See, for example, [1, 5, 8]. Here we give a probabilistic analysis of (1), under the assumption that all $a_{i j}, c_{i j}$ are independently and uniformly distributed in $[0,1]$. We examine the asymptotic behaviour as $n \rightarrow \infty$, while $m$ and the $\beta_{i}$ remain fixed. (The assumption that $m$ is constant while $n \rightarrow \infty$ is realistic, for example, in the machine-scheduling application.) We assume $m>1$, since otherwise the problem is obviously trivial.

We have chosen the maximising form for (1). All our results apply equally well to the minimising form, since

\footnotetext{
*Supported by NATO grant RG0088/89

${ }^{\dagger}$ Supported by NSF grant CCR-8900112 and NATO grant RG0088/89
} 
the constraints imply that the objective function

$$
z=n-\sum_{i=1}^{m} \sum_{j=1}^{n}\left(1-c_{i j}\right) x_{i j} .
$$

and $\left(1-c_{i j}\right)$ has precisely the same distribution as $c_{i j}$.

The work presented here is closely related to, and in part rests on, our earlier paper [2] on the multidimensional knapsack problem. (This was itself an extension of work of Lueker [6] and Goldberg and MarchettiSpaccamela [3].) As in that paper, the main technique here is to relate the solution of the integer program (1) to its linear programming (LP) relaxation:

$$
\text { (1) with } x_{i j} \in\{0,1\} \text { replaced by } x_{i j} \geq 0 \text {. }
$$

As in [2], we use strongly the fact that the only conditioning on the data imposed by the solution of (2) arises from satisfying the primal and dual feasibility conditions (which are simply linear inequalities). We make use of well-known ideas from LP theory throughout the paper without comment. We use similarly standard methods from probability theory, in particular certain probability inequalities. See [7] for a useful survey of many such inequalities.

However, although our methods are similar to those of [2], extending a probabilistic analysis to an apparently similar problem is not straightforward, since changed structure produces different conditioning. Nevertheless, we will show that in this case the difficulties can be successfully negotiated. There is one proviso. For technical reasons, we must assume the following condition on the $\beta_{i}$ holds. Consider the vector $\beta$ as a point in $\boldsymbol{R}^{m}$. Let the set $\mathcal{F}$ be (the convex set of) those $\beta$ for which (2) remains feasible with probability tending to 1 as $n \rightarrow \infty$. We clearly need $\beta \in \mathcal{F}$ in order that the feasibility of (1) does not dominate the analysis. We assume something stronger, however. Let $\mathcal{F}(\eta)$ be the set of $\beta$ such that (2) remains feasible (with probability tending to 1 ) when the $\beta_{i}$ are (all) reduced to $\beta_{i}-\eta$, for given $\eta>0$. If $\beta \in \mathcal{F}(\eta)$, we will call (1) (or (2) ) $\eta$-feasible. We require $\eta$-feasibility for some $\eta>0$. It is clear that int $\mathcal{F}=\bigcup_{\eta>0} \mathcal{F}(\eta)$. Thus, in a certain sense, our analysis covers "almost all" $\beta$ 's of interest.

From an algorithmic viewpoint, we do not require conditions for $\eta$-feasibility (for some $\eta>0$ ). However, various simple sufficient conditions can be derived. For example,

$$
\eta=\min _{1 \leq i \leq m} \beta_{i}-\frac{1}{m(m+1)}>0
$$

is such a condition. To see this, consider the solution to (2) given by setting $x_{i j}=1$ in column $j$ for the $i$ which gives the minimum $a_{i j}$ in that column. Since this minimum is $1 /(m+1)$ in expectation (the minimum of $m$ uniform [0,1]'s), and will occur in each row with probability $1 / m$, the sufficiency of the condition follows easily from the law of large numbers.

The results we prove are specifically as follows. The first relates the objective values of (1) and (2). (Natural logarithms are used throughout.) 
Theorem 1 Let (1) be $\eta$-feasible for some $\eta>0$, and $z_{I P}, z_{L P}$ be the optimal values for (1), (2) respectively. Then there exist constants $0<p(m)<1$ and $\alpha(m, \eta)>0$ such that, for large $n$,

$$
\begin{aligned}
\operatorname{Pr}\left(z_{L P}-z_{I P}>t \alpha(\log n)^{2} / n\right) & <p^{t}, \quad\left(t=1,2, \ldots,\left\lfloor n /(\log n)^{2}\right\rfloor\right) \\
\text { and } E\left(z_{L P}-z_{I P}\right) & <\left(2 \alpha /(1-p)^{2}\right)(\log n)^{2} / n .
\end{aligned}
$$

Moreover, if $\mathcal{N}$ is the number of variables whose values differ in the two optimal solutions, then there exists $\sigma(m, \eta)$ such that

$$
E(\mathcal{N})<\sigma \log n
$$

The second shows that, with any specified probability of error, (1) can be solved in polynomial time.

Theorem 2 Let (1) be $\eta$-feasible for some $\eta>0$, and $\tau>0$ a probability. Then there exists a constant $d(m, \eta, \tau)$, such that a proven optimal solution can be obtained by a branch-and-bound search algorithm in time $O\left(n^{d}\right)$ with probability at least $(1-\tau)$.

(Unfortunately, our estimate for $d$ grows quite rapidly as $\tau \rightarrow 0$.)

The plan of the paper is as follows. In $\S 2$ we give some preliminary results on the linear programming (LP) relaxation of (1). In $\S 3$ we prove Theorem 1 and in $\S 4$ we prove Theorem 2. Finally $\S 6$ gives some brief concluding remarks.

\section{Linear Programming Preliminaries}

We show here some simple properties of the LP problem (2) and its dual:

$$
\begin{aligned}
\text { Minimise } & \sum_{i=1}^{m} b_{i} u_{i}+\sum_{j=1}^{n} v_{j} \\
\text { subject to } & a_{i j} u_{i}+v_{j} \geq c_{i j} \quad(\forall i, j) \\
u_{i} & \geq 0 \quad(\forall i) .
\end{aligned}
$$

Lemma 3 below has been given by Benders and Vannunen [1], with a different proof and a slightly weaker conclusion.

Lemma 3 Let $\left\{x_{i j}^{B}\right\}$ be any basic feasible solution to the system

$$
\begin{array}{ll}
\text { subject to } & \sum_{j=1}^{n} a_{i j} x_{i j} \leq b_{i} \quad(i=1,2, \ldots, m) \\
& \sum_{i=1}^{m} x_{i j}=1 \quad(j=1,2, \ldots, n) \\
\text { and all } & x_{i j} \geq 0 .
\end{array}
$$

and let $F^{B}=\left\{(i, j): 0<x_{i j}^{B}<1\right\}, E^{B}=\left\{j: \exists(i, j) \in F^{B}\right\}$. Then (a) $\left|E^{B}\right| \leq m$, and (b) $\left|F^{B}\right| \leq 2 m$. 
Proof Any basic feasible solution to (4) is an optimal solution for some objective function

$$
\text { Maximise } z=\sum_{i=1}^{n} \sum_{j=1}^{n} \hat{c}_{i j} x_{i j} \text {. }
$$

In such a solution, for each $j$, there is an $i^{*}(j)$ such that $x_{i^{*} j}>0$. We use the constraints $\sum_{i=1}^{m} x_{i j}=1$ to eliminate the $x_{i^{*} j}$. This will give an LP:

$$
\begin{array}{ll}
\text { Maximise } & z=\sum_{j=1}^{n} \sum_{i \neq i^{*}} c_{i j}^{\prime} x_{i j} \\
\text { subject to } & \sum_{j=1}^{n} \sum_{i \neq i^{*}} a_{i j k}^{\prime} x_{i j} \leq b_{k}^{\prime} \quad(k=1,2, \ldots, m) \\
& \sum_{i \neq i^{*}} x_{i j} \leq 1 \quad(j=1,2, \ldots, n) \\
\text { and all } & x_{i j} \geq 0 .
\end{array}
$$

Now in the LP (5), all constraints $\sum_{i \neq i^{*}} x_{i j} \leq 1$ are non-binding at the optimum, and hence redundant. Thus the solution will be the same as that to

$$
\begin{array}{ll}
\text { Maximise } & z=\sum_{j=1}^{n} \sum_{i \neq i^{*}} c_{i j}^{\prime} x_{i j} \\
\text { subject to } & \sum_{j=1}^{n} \sum_{i \neq i^{*}} a_{i j k}^{\prime} x_{i j} \leq b_{k}^{\prime} \quad(k=1,2, \ldots, m) \\
\text { and all } & x_{i j} \geq 0 .
\end{array}
$$

Let $F=\left\{(i, j): x_{i j}>0\right.$ in the optimal solution to (5) $\}$ and $E=\{j: \exists(i, j) \in F\}$. Clearly $|E| \leq|F|$, and since there is an optimal basic solution, $|F| \leq m$. Now $x_{i^{*} j}<1$ if and only if $j \in E$. Thus $E^{B}=E$, proving (a). Also $F^{B}=F \cup\left\{\left(i^{*}, j\right): j \in E\right\}$, and since the union is disjoint $\left|F^{B}\right|=|F|+|E| \leq 2 m$, proving (b).

Lemma 4 Any basic feasible solution to (3) can be completely specified by giving $E^{B} \subseteq\{1,2, \ldots, n\}$ with $\left|E^{B}\right| \leq m$, and $F^{B} \subseteq\left\{(i, j): j \in E^{B}\right\}$ with $\left|F^{B}\right| \leq 2 m$.

Proof Consider the dual (3). We assume, without loss of generality, that it is non-degenerate. If the set $F^{B}$ is as defined in Lemma 3 , then the set of equations

$$
a_{i j} u_{i}+v_{j}=c_{j} \quad(i, j) \in F^{B}
$$

will uniquely determine the non-zero $u_{i}$. The remaining $v_{j}$ can now be determined by $v_{j}=\max _{i}\left\{c_{i j}-a_{i j} u_{i}\right\}$. This completely specifies the dual basic feasible solution, and the primal solution can be recovered by complementary slackness.

The method of specifying a dual basic feasible solution implied by Lemma 4 is central to our arguments below. It has the following important consequence.

Corollary 5 (Assuming non-degeneracy) there are less than $(6 m)^{m} n^{m}$ basic feasible solutions to (3).

Proof There are clearly at most

$$
\left(\begin{array}{c}
n \\
m
\end{array}\right)\left(\begin{array}{l}
m^{2} \\
2 m
\end{array}\right)<(e n / m)^{m}\left(e m^{2} / 2 m\right)^{2 m}=\left(e^{3} m n / 4\right)^{m}<(6 m n)^{m}
$$


ways of selecting $E^{B}$ then $F^{B}$.

The non-degeneracy assumption holds with probability 1 in our model. Thus, as $n \rightarrow \infty$ with $m$ fixed, we will have $O\left(n^{m}\right)$ (i.e. a polynomial number of) dual basic feasible solutions to consider. This should be contrasted with the $m^{n}$ (i.e. an exponential number of) potential primal basic feasible solutions.

We now show that the $\eta$-feasibility condition discussed in $\S 1$ implies a bound on the optimal dual variables in (2). For ease of notation let us write (2) in "matrix form", i.e.

$$
\begin{gathered}
L P(b): \quad z_{L P}(b)=\max z \\
\text { subject to } z=c x, \quad A x \leq b, \quad E x \leq e_{n}, \quad x \geq 0 .
\end{gathered}
$$

where, in particular, $e_{n}$ denotes an $n$-vector of 1's.

Lemma 6 Let $\eta>0$. If (2) is $\eta$-feasible and $u_{i}(i=1, \ldots, m)$ are the dual variables in its optimal solution, then

$$
\sum_{i=1}^{n} u_{i} \leq 1 / \eta
$$

Proof Let $x$ be any feasible solution to $L P\left(b-n \eta e_{m}\right)$. Clearly $c x \geq 0$. The optimal dual variables for $L P(b)$ satisfy

$$
u A+v E \geq c, \quad u \geq 0, \quad u b+v e_{n} \leq n,
$$

the last inequality following from the duality theorem and the obvious upper bound on the primal objective function. Hence

$$
\begin{aligned}
n & \geq u b+v e_{n} \\
& \geq u\left(A x+\eta n e_{m}\right)+v e_{n} \\
& =u A x+\eta n u e_{m}+v E x \\
& =(u A+v E) x+\eta n u e_{m} \\
& \geq c x+\text { qnue }_{m} \\
& \geq \text { qnue }_{m} .
\end{aligned}
$$

Thus $u e_{m} \leq 1 / \eta$, as required.

The last result in this section relates the optimal solution of (2) to "nearly optimal" solutions when the right hand sides are changed.

Lemma 7 Let $y^{\prime}$ be an optimum basic feasible solution to $L P\left(b^{\prime}\right)$ and $x=y^{\prime}+\theta$ any feasible solution to $L P(b)$. Let $u \geq 0$ be the optimum dual variables for the inequalities in $L P\left(b^{\prime}\right)$ and $\bar{c} \geq 0$ the reduced costs associated with the nonbasic variables in $y^{\prime}$. Then

$$
c x \leq z_{L P}(b)+\bar{c} \theta+u(b-A x)
$$


Proof From the objective row of the basis associated with $y^{\prime}$ we have

$$
c x=\bar{c} x+u\left(b^{\prime}-A x\right)+c y^{\prime} .
$$

Let $y \geq 0$ solve $L P(b)$, so

$$
z_{L P}(b)=c y=\bar{c} y+u\left(b^{\prime}-A y\right)+c y^{\prime}
$$

Thus

$$
\begin{aligned}
c x-c y & =\bar{c}\left(y^{\prime}+\theta\right)-\bar{c} y+u(A y-A x) & & \\
& =\bar{c} \theta-\bar{c} y+u(A y-A x) & & \left(\bar{c} y^{\prime}=0\right) \\
& \leq \bar{c} \theta+u(A y-A x) & & (\bar{c} \geq 0, y \geq 0) \\
& \leq \bar{c} \theta+u(b-A x) & & (A y \leq b, u \geq 0)
\end{aligned}
$$

\section{Proof of Theorem 1}

Assume $1>\eta>0$ is given, and that (1) is $\eta$-feasible. Let $K=\frac{9}{20} m^{2} \eta \log n, k=\left\lfloor 18 m^{2} \log n\right\rfloor, M=2^{m+9} / \eta^{2}$ and $\epsilon=80 m^{3} M \log n / n$.

Now, for $i=1, \ldots, m$, let $b_{i}^{\prime}=b_{i}-K$. Let $x^{B}$ solve $L P\left(b^{\prime}\right)$. From Lemma 6 the dual variables $u_{i}$ for $L P\left(b^{\prime}\right)$ satisfy

$$
\sum_{i=1}^{m} u_{i} \leq \frac{1}{\eta-K / n}<2 / \eta
$$

for all $n$ large enough. So $u_{i}<2 / \eta$, for all $i$.

Let $E^{B}, F^{B}$ be as defined in $\S 2$. For each $j=1, \ldots, n$ define $i^{*}(j)$ as follows. For $j \notin E^{B}$, let $i^{*}(j)$ be the (unique) $i$ such that $x_{i j}^{B}=1$. For $j \in E^{B}$, choose $i^{*}(j)$ arbitrarily from $\left\{i:(i, j) \in F^{B}\right\}$. We define a "rounded" zero-one solution $\xi^{B}$ by

$$
\xi_{i j}^{B}=1 \text { if and only if }(i, j)=\left(i^{*}, j\right) .
$$

Now let

$$
\begin{aligned}
\Delta_{i}^{B} & =b_{i}-\sum_{j=1}^{n} a_{i j} \xi_{i j}^{B} & & \left(u_{i} \neq 0\right) \\
& =K & & \text { otherwise }
\end{aligned}
$$

Then, since $0 \leq a_{i j} \leq 1$,

$$
K-m \leq \Delta_{i}^{B} \leq K+m .
$$

We now wish to "fill the deficits" $\Delta_{i}^{B}$ by changing only the values (in $\xi^{B}$ ) of variables with small reduced costs. Then we will use Lemma 7 to show that the solution $\xi$ so constructed is close to optimal. We change the $\xi^{B}$ by making cyclic changes within chosen blocks of $m$ columns. To this end, we make the following definitions. Let $s$ be any integer. We define an $s$-good block to be a set of $m$ columns $j$, as follows. For ease of notation, let us assume the block comprises columns $1,2, \ldots, m$, and that subscript arithmetic is modulo $m$. Let us write $\tilde{c}_{i j}=c_{i j}-u_{i} a_{i j}$. Then the block must be such that, for $1,2, \ldots, m$ 
(a) $\xi_{i i}^{B}=1$. (The changes we intend to make are $\xi_{i i}=0, \xi_{i-1, i}=1$.)

(b) $a_{i i} \leq \eta / 20, a_{i, i+1} \in\left[a_{i i}, a_{i i}+\eta / 20\right]$.

(c) $c_{j i} \leq \frac{1}{2}, j \neq i, i-1, \frac{3}{5}<c_{i i} \leq \frac{4}{5}$, and

$$
c_{i-1, i} \in\left[\tilde{c}_{i i}+u_{i-1} a_{i-1, i}-s \epsilon, \tilde{c}_{i i}+u_{i-1} a_{i-1, i}-(s-1) \epsilon\right]
$$

These imply, by straightforward calculations, the following conditions for column $i$ in the block,

(i) $\tilde{c}_{k i} \leq c_{k i} \leq \frac{1}{2}$ for $k \neq i, i-1$. (The LP solution must not pick $x_{k i}$.)

(ii) $\quad \tilde{c}_{i i}>\frac{3}{5}-\frac{1}{10}=\frac{1}{2}$. (The LP solution must pick $x_{i i}$.)

(iii) $(s-1) \epsilon \leq \tilde{c}_{i-1, i}-\tilde{c}_{i, i} \leq s \epsilon$. (The LP solution picks $x_{i i}$, but $x_{i-1, i}$ is "nearly as good". Observe also that conditions (i)-(iii) imply that $\bar{c}_{i j}=\tilde{c}_{i j}-\tilde{c}_{i i}$ for all $i, j$.)

(iv) $a_{i, i+1}-a_{i i}$ is uniform in $[0, \eta / 20]$ independently, even conditional on $a_{i i}$. (We need this since we wish to argue relative to the optimal basis for $L P\left(b^{\prime}\right)$, and $a_{i i}$ is then conditioned by feasibility of the solution.)

(v) $\left[\tilde{c}_{i i}+u_{i-1} a_{i-1, i}-s \epsilon, \tilde{c}_{i i}+u_{i-1} a_{i-1, i}-(s-1) \epsilon\right] \subseteq[0,1]$ for $s \leq 1 / 2 \epsilon$. (We require that the probability that $c_{i-1, i}$ falls in this range depends only on its width, and not its location.)

Now the probability that a given column $j$ can be the $i$ th column in an $s$-good block is clearly a priori at least

$$
\left(\frac{1}{2}\right)^{m-2} \times \frac{1}{5} \times\left(\frac{\eta}{20}\right)^{2} \times \epsilon>\frac{\epsilon}{M},
$$

independently of $i$ or $j$.

Suppose now that, for any dual feasible basis $D$, we try to construct $s$-good blocks. The determination of the $u_{i}$ conditions only $m$ columns, by Lemma 4 . We now scan the remaining $(n-m)$ columns from left to right, attempting to form good blocks one column at a time. There is one problem. The circularity of our conditions for the columns of the blocks implies that we cannot test column 1 in the block until column $m$ is known. For this reason we have to proceed slightly more carefully, as follows. We denote the elements of the columns as in the above conditions, but the reader should observe that we are in fact examining a general column $j$ for potential addition to the current incomplete block. We scan to select the first column, checking conditions (a)-(c). However, instead of checking condition (b) on $a_{i-1, i}$ (i.e. $a_{m 1}$ ) (since this is currently impossible) we merely check the necessary condition that $a_{m 1} \leq \eta / 10$. Note that we also check condition (c) involving $a_{m 1}$, but the choice of our ranges implies that this does not further condition $a_{m 1}$. It merely locates the point $\left(c_{m 1}, a_{m 1}\right)$ in a small parallelogram whose projection is uniform on the $a_{m 1}$-axis. Having chosen column 1 we scan to add columns $2, \ldots, m-1$, in each case checking conditions (a)-(c). There is no difficulty since the required quantities are all known at the time of checking. We now select column $m$. First 
we check all conditions except that in (b) involving $a_{m 1}$. We will check condition (c) involving $a_{m m}$, but this does not condition it further than $a_{m m} \leq \eta / 20$, by the same argument used for $a_{m 1}$. Let us call a "block" which has passed all tests up to this point a candidate block. Now the point $\pi=\left(a_{m m}, a_{m 1}\right)$ is uniformly distributed in the rectangle $\Pi=[0, \eta / 20] \times[0, \eta / 10]$. We now test condition (b) on $a_{m 1}$. This requires that the point $\pi$ lies in a parallelogram whose area is half that of $\Pi$. Thus the probability that a candidate block is $s$-good is $\frac{1}{2}$. If the candidate block is not good, we reject it completely and start afresh. This may appear drastic, but to do otherwise introduces extra conditioning, and the above line of argument fails.

Note that, as we form candidate blocks, the probability of a given column being successfully added is still (independently) at least $\epsilon / M$, since we check some subset of (a)-(c). However some candidate blocks will not be good. Now the expected number of successful additions is $(n-m) \epsilon / M>4 m k$ for large $n$. Thus

$$
\begin{aligned}
\operatorname{Pr}(\text { Under } 3 k \text { candidates formed }) & =\operatorname{Pr}(\text { Under } 3 k m \text { successful additions }) \\
& <e^{-\frac{1}{8} m k} \\
& <n^{-2 m^{3}}
\end{aligned}
$$

for large $n$, using Hoeffding's inequality $[4,7]$. Then, since each candidate is good with probability $\frac{1}{2}$.

$$
\begin{aligned}
\operatorname{Pr}(\text { Under } k s \text {-good blocks formed }) & <e^{-k / 18}+n^{-2 m^{3}} \\
& <2 n^{-m^{2}}
\end{aligned}
$$

for large $n$, again using Hoeffding's inequality.

Now, since there are less than $n$ possible values of $s$, and only $O\left(n^{m}\right)$ dual feasible bases $D$ to consider, we see that for large $n$,

$$
\operatorname{Pr}(\exists D, s \text { : less than } k s \text {-good blocks exist })<n^{-\frac{1}{2} m^{2}} .
$$

For a given $s$-good block, let $\delta_{i}=a_{i, i-1}-a_{i i}$ for $i=1,2, \ldots, m$. These give the changes in the left hand sides of the constraints in (1) when the cyclic changes $\xi_{i i}=0, \xi_{i-1, i}=1$ are made to the variables in the block. If we have $k s$ - good blocks we denote the corresponding values by $\delta_{i r}$ for $r=1,2, \ldots, k$. By construction the $\delta_{i r}$ are distributed uniformly and independently in $[0, \eta / 20]$. Let

$$
Z_{i r}=\left(\frac{\delta_{i r}-\eta / 40}{\eta / 40}\right) \sqrt{3}
$$

$Z_{\text {ir }}$ is uniform in $[-\sqrt{3},+\sqrt{3}]$ with mean 0 , variance 1 . Thus

$$
\begin{aligned}
\Delta_{i}^{B}-\sum_{r=1}^{k} \delta_{i r} & =\Delta_{i}^{B}-\left(\frac{\eta}{40 \sqrt{3}}\right) \sum_{r=1}^{k} Z_{i r}-\frac{k \eta}{40} \\
& =\Gamma_{i}-\frac{\eta}{40 \sqrt{3}} \sum_{r=1}^{k} Z_{i r},
\end{aligned}
$$


where $\Gamma_{i}=\Delta_{i}^{B}-(k \eta / 40)=K-(k \eta / 40)+O(1)=O(1)$, using (8) and the definitions of $k, K$. Let $\Phi_{i}=40 \sqrt{3} \Gamma_{i} / \eta$. Now we use the following Lemma, which is a (modified) restatement of Lemma 3.4 of [2].

Lemma 8 Let $Z_{i r}, i=1, \ldots, m, r=1, \ldots, k$ be bounded i.i.d. random variables with bounded density, expectation 0 and variance 1. Let $I_{i}=\left[\Phi_{i}-\theta, \Phi_{i}\right]$, where $\theta=\frac{(\pi k / 2)^{(m+1) / 2 m}}{2^{k / m+1 / 2}}$ and $\Phi_{i}=O\left(k^{1 / 4}\right)$, be $m$ intervals. Let $q_{m}=\frac{1}{2\left(1+(2 / \sqrt{3})^{m}\right)}$, and let $S$ be an arbitrary subset of $\{1,2, \ldots, k\}$ with $|S|=\lfloor k / 2\rfloor$. Then, for large $k$,

$$
\operatorname{Pr}\left(\exists S: \sum_{r \in S} Z_{i r} \in I_{i}, i=1, \ldots, m\right) \geq q_{m} .
$$

Applying this to our situation, we see that with probability at least $q_{m}$ we can choose $\lfloor k / 2\rfloor s$-good blocks to change which will leave the deficit in each left hand side in (1) at most $\eta \theta / 40 \sqrt{3}$. Substituting for $k$, this is less than (say) $n^{-10 m}$, for large $n$. Now apply Lemma 7 . By using only $s$-good blocks, with probability at least $q_{m}$ we can construct an integer solution $\xi$ to (1) with objective value $z_{I P}$ satisfying

$$
0 \leq z_{L P}-z_{I P} \leq k s \epsilon+2 m n^{-10 m} / \eta<s \alpha(\log n)^{2} / n,
$$

for large $n$, with $\alpha=2^{m+20} m^{5} / \eta^{2}$, say. Now we try the $s$-good sets for $s=1,2, \ldots, t$, seeking to find $s$ so that (10) is satisfied. (Note that $t$ can be as large as $n /(\log n)^{2}$.) Let $p=1-q_{m}$. Each "trial" is independent (since it involves only the $\delta_{i r}$ ). The probability that we fail $t$ times is thus at most $p^{t}$, as required. This proves the first statement of Theorem 1. The second is derived from it as follows.

Let $t=\lceil 2 \log n / \log (1 / p)\rceil$, say. Using a simple bound on expectation, and the fact that $z_{L P}-z_{I P} \leq n$ always, we have

$$
\begin{aligned}
0 \leq E\left(z_{L P}-z_{I P}\right) & \leq\left(\alpha(\log n)^{2} / n\right) \sum_{s=1}^{t} s p^{s-1}+n p^{t} \\
& <\left(\alpha /(1-p)^{2}\right)(\log n)^{2} / n+1 / n \\
& <\left(2 \alpha /(1-p)^{2}\right)(\log n)^{2} / n
\end{aligned}
$$

for large enough $n$. The final statement of Theorem 1 follows from this and Lemma 9 below. Let

$$
C_{1}=\left\{(i, j): \bar{c}_{i j} \in(0,2 \log n / n]\right\}
$$

It is shown in Lemma 9 that

$$
E\left(\left|C_{1}\right|\right)<4 m^{2} \log n .
$$

Now, there are at most $2 m$ fractional basic variables by Lemma 3. Thus clearly

$$
\mathcal{N} \leq 2 m+\left|C_{1}\right|+\frac{\left(z_{L P}-z_{I P}\right)}{2 \log n / n} .
$$

Taking expectations of both sides in this inequality, the theorem now follows using (11) and (12). 


\section{Proof of Theorem 2}

Let $\eta>0$ be given, and let $0 \leq \tau \leq 1$ be the required failure probability. Assume $n$ is large enough that

$$
\operatorname{Pr}((1) \text { is } \eta \text {-feasible }) \geq 1-\tau / 2 \text {. }
$$

Now consider the following algorithm.

\section{Algorithm $O P T_{\tau}$}

(1) $t \leftarrow\lceil\log (\tau / 2) / \log p\rceil$.

(2) Solve $L P(b)$ giving $x^{B}$. Modify this to $\xi^{B}$.

(3) Let $T=\left\{(i, j): \xi_{i j}^{B}=0,0 \leq \bar{c}_{i j} \leq t \alpha(\log n)^{2} / n\right\}$, where $\bar{c}_{i j}$ are the reduced costs for $x^{B}$.

(4) Compute $\min _{S \subseteq T} c \xi_{S}$, for all $S$ and $\xi_{S}$ such that

(a) there is at most one $(i, j) \in S$ for all $j$.

(b) $\sum_{(i, j) \in S} \bar{c}_{i j} \leq t \alpha(\log n)^{2} / n$.

(c) $\xi_{S}$ is feasible in $(1)$, where $\xi_{S}$ is obtained from $\xi^{B}$ by $\xi_{i j} \leftarrow 1, \xi_{k j} \leftarrow 0(k \neq i)$, for each $(i, j) \in S$.

The minimising $S$ at termination (if defined), gives an optimal solution $\xi_{0}=\xi_{S}$ to (1). This follows directly, since the search accounts for all feasible integer solutions having objective value at $\operatorname{most} z_{L P}+t \alpha(\log n)^{2} / n$. Failure because (1) is not $\eta$-feasible has probability at most $\tau / 2$. The probability that $S$ is undefined is, by Theorem 1 , less than $p^{t}$, which is at most $\tau / 2$ by choice of $t$. Thus it remains to analyse the running time of $O P T_{\tau}$.

Fix any dual feasible basis $D$, and let

$$
\begin{aligned}
& J_{l}=(2(l-1) \log n / n, 2 l \log n / n], \\
& C_{l}=\left\{(i, j): \bar{c}_{i j} \in J_{l}\right\} .
\end{aligned}
$$

$\left(C_{1}\right.$ has already been used in $\S 3$. $)$

Lemma $9 \operatorname{Pr}\left(\left|C_{l}\right| \geq 4 m^{2} \log n\right) \leq n^{-2 m^{2} / 3}$.

Proof For any column $j$ not determining the $u_{i}$, we have

$$
\begin{aligned}
E\left(\text { number of }(i, j) \in C_{l}\right) \leq & E\left(\text { number of pairs } i, r: \tilde{c}_{i j}-\tilde{c}_{r j} \in J_{l}\right) \\
\leq & m(m-1) \operatorname{Pr}\left(c_{1 j} \in[\lambda, \lambda+2 \log n / n]\right), \\
& \quad \text { for } \lambda=u_{1} a_{1 j}+c_{2 j}-u_{2} a_{2 j}, \\
\leq & 2 m^{2} \log n / n .
\end{aligned}
$$


Hence, using Hoeffding's inequality,

$$
\operatorname{Pr}\left(\left|C_{l}\right|>4 m^{2} \log n\right) \leq e^{-2 m^{2} \log n / 3}=n^{-2 m^{2} / 3} .
$$

Since there $O\left(n^{m}\right)$ dual feasible bases, and we are interested in only $O(\log n)$ values of $l$, it follows from Lemma 9 that we may assume $\left|C_{l}\right| \leq 4 m^{2} \log n$ for all $l$ in the optimal basis. We examine the search in $O P T_{\tau}$ relative to the sets $C_{l}$. To these we have only to add the (less than $2 m$ by Lemma 3 ) fractional variables which have been rounded down. The argument is now similar to that in [2].

Thus let $R_{r}=\bigcup_{l=2^{r-1}}^{2^{r}-1} C_{l}(r=1,2, \ldots)$. We clearly need only consider $S$ for which

$$
\left|S \cap R_{r}\right| \leq\left\lfloor\frac{t \alpha(\log n)^{2} / n}{\left(2^{r}-2\right) \log n / n}\right\rfloor=\left\lfloor\frac{t \alpha \log n}{\left(2^{r}-2\right)}\right\rfloor=\rho_{r},
$$

say, if $r>1$. Note that $\rho_{r}=0$ for all large enough $r$. Thus, with probability $1-o(1)$, we need to check at most $N$ sets $S$, where

$$
N=2^{2 m} \times 2^{4 m^{2} \log n} \times \prod_{r=2}^{\infty} N_{r}
$$

sets $S$, where

$$
N_{r}=\sum_{r=0}^{\rho_{r}}\left(\begin{array}{c}
\left\lfloor 4 m^{2} \log n 2^{r-1}\right\rfloor \\
r
\end{array}\right) .
$$

Now define $r_{0}=\max \left\{r: \frac{t \alpha}{2^{r}-2} \geq m^{2} 2^{r-1}\right\}$, and note that $r_{0}$ is independent of $n$. For all $r$, clearly $N_{r} \leq 2^{4 m^{2} \log n 2^{r-1}}$. However, for $r \geq r_{0}$, using well-known bounds on binomial coefficients, we have the better bound

$$
N_{r} \leq 2\left(\frac{e 4 m^{2} \log n 2^{r-1}}{\rho_{r}}\right)^{\rho_{r}} \leq 2^{\gamma\left(r / 2^{r}\right) \log n},
$$

after a straightforward calculation, for large $n$ and suitably chosen $\gamma$ (independent of $n$ and $r$ ). Hence

$$
N \leq 2^{2 m+4 m^{2} \log n+\sum_{r=2}^{r_{0}} 2^{r+1} m^{2} \log n+\sum_{r=r_{0}+1}^{\infty} \gamma\left(r / 2^{r}\right) \log n}=n^{d-1},
$$

for a suitable $d$. Since each set $S$ takes $O(n)$ time to process, the theorem follows.

\section{Extension to More General Distributions}

The preceding analysis can be extended quite easily to more general distributions. We can assume for example that the $c_{i, j}$ are drawn independently from one distribution $F_{c}$ and the $a_{i, j}$ are drawn independently from another $F_{a}$. These distributions should have bounded supports $\left[0, L_{c}\right],\left[0, L_{a}\right]$ respectively and positive densities throughout. Under these circumstances our results go through with only marginal changes. Thus the inequality (9) will have a right hand side $\epsilon / M^{\prime}$ where $M^{\prime}$ depends on $m, \eta$ and $F_{a}$. As in the case of 
the uniform distribution, we will be able to show the existence of sufficiently many $s$-good blocks with high probability. We can then use Lemma 8 to finish the proof of the theorem, since it handles sufficiently general random variables. The proof of Theorem 2 will not change much either, since Lemma 9 will require little modification. The same comments seem to be true for distributions which have positive density on $[0, \infty)$ and small enough tail probabilities, e.g. the exponential distribution.

If the distributions are discrete, then our analysis does not go through so easily. However, we imagine that, for $c_{i, j}$ uniform in $\left\{0,1, \ldots, L_{c}\right\}$ and $a_{i, j}$ uniform in $\left\{0,1, \ldots, L_{a}\right\}$ say, it may not be too difficult to modify our proof, provided $L_{c}$ and $L_{a}$ grow sufficiently fast with $n$.

Other types of distribution might pose more severe problems and are left to future research.

\section{Final Remarks}

(1) For convenience in the development, we have stated all our results for large $n$, but obviously they can be modified to hold for all $n$ (simply by enlarging the constants if necessary).

(2) We claim only that our results hold for constant $m$, but it would appear that generally they can be modified to remain true if $m$ grows slowly enough with $n$. However, the running time is (at least) $\Omega\left(C^{m^{2} \log n}\right)$, for some constant $C$. Thus the algorithm is not provably polynomial time for non-constant $m$.

(3) The approach we have developed here appears to be applicable to some related problems, for example the multiple-choice multidimensional knapsack problem.

\section{References}

[1] J. F. Benders and J. A. Vannunen, "A property of assignment type mixed integer programs", Operations Research Letters, (1983) 47-52.

[2] M. E. Dyer and A. M. Frieze, "Probabilistic analysis of the multidimensional knapsack problem", Mathematics of Operations Research, (1989) 162-176.

[3] A.V.Goldberg and A.Marchetti-Spaccamela, "On finding the exact solution of a zero-one knapsack problem", in Proceedings of the 16th Annual ACM Symposium on the Theory of Computing, (ACM Press 1984) 359-368.

[4] W.Hoeffding, "Probability inequalities for sums of bounded random variables", Journal of the American Statistical Association (1963) 13-30. 
[5] K.O.Jörnsten and M.Nasberg, "A new Lagrangian relaxation approach to the generalised assignment problem", European Journal of Operations Research (1986) 313-323.

[6] G.S.Lueker, "On the average difference between the solutions to linear and integer knapsack problems", in: R.L.Disney and T.J.Ott eds., Applied Probability-Computer Science: The Interface, Vol. I, Progress in Computer Science, (Birkhäuser, Boston, 1982) pp.489-504.

[7] C.J.H.McDiarmid, "On the method of bounded differences, in Surveys in Combinatorics, J. Siemons ed., London Mathematical Society Lecture Notes 141, 1989, pp.148-188.

[8] G. T. Ross and R. M. Soland, "A branch-and-bound algorithm for the generalised assignment problem", Mathematical Programming (1975) 91-103. 